\title{
ON HOMOGENEOUS OSCILLATION OF SUCCESSIONS OF
}

\section{FUNCTIONS}

\author{
$B y$. W. H. Youna, Sc.D., F.R.S.
}

[Received December 15th, 1909.-_Read January 13th, 1910.]

1. In considering successions of functions which oscillate, we may either require the property which we are discussing to be true of the succession as a whole, or we may require it also to be true for every succession contained in the original succession, say of every sub-succession. or sub-set. Thus uniform oscillation has been defined* with respect to the whole succession, while Arzela's celebrated postulate of equal continuity from its very nature applies to every sub-succession. The theorem t of Arzela's which gives the result of this postulate of equal continuity has recently attracted so much notice that it has seemed to me worth while to shew how it follows naturally from the theory of uniform oscillation, as developed by myself. I am thus led to give a number of theorems which may perhaps from their simplicity, as well as from the light they throw on the theorem of Arzeli, be worthy of notice.

I nse the word "homogeneous" in connection with an oscillation to denote that the property under consideration is possessed not only by the succession itself, but by every one of its sub-successions. By the upper. (lower) function of a succession I denote the function whose value at every point is the upper (lower) limit of the succession at that point. This is the upper (lower) function par excellence; when we are considering the succession as the ensemble of all its sub-successions, we shall use the expression all the upper and all the lower functions of the succession to denote all the upper and lower functions of every sub-succession. When the upper and lower function of a succession, or sub-succession, coincide, each will be called the unique limiting function $\ddagger$ of the succession, or subsuccession, and such a succession, or sub-succession, will be called a sequence.

" "On Oscillating Successions of Continuous Functions." Proc. London Math. Suc., Ser. 2, Vol. 6, pp. 298-320.

† C. Arzelà, "Sulle Funzioni di Linee," 1895, Mem. della R. Acc. delle Scicnze dell' Ist. di Bologna, Serie 5, Vol. v, pp. 3-22. It is no longer necessary to emphasise the importance of the theorem in question. It has been found to be of use not only in the theory of integrals and of differential equations and of infinite series, but also in the calculus of variations, as well as in more abstract speculations.

$\ddagger$ This usage is different from that of Arzeli.

SER. 2. VOL. 8. No. 1058 . 
The main results arrived at are as follows :-

If all the lower functions of a succession are lower semi-continuous on the right (left), so are the upper; and if all the upper functions are upper semi-continuous on the right (left), so are the lower.

If all the upper, or all the lower, functions are continuous on the right (left), then a sequence of the functions can be found, having an unique limiting function which is continuous on the right (left).

If the succession of continuous functions oscillates uniformly and homogeneously, then there is a sequence of the functions which converges uniformly to a continuous limiting function, and all the upper as well as all the lower functions of the succession are continuous.

More generally if a succession of continuous functions oscillates uniformly and homogeneously on the right (left), then all the upper and all the lower functions are continuous on the right (left), and in every subsuccession there is a sequence of the functions, converging towards an unique limiting function which is continuous on the right (left), the convergence to this limiting function being, moreover, uniform on the right (left).

As a corollary to this last theorem we have a theorem which includes Arzela's celebrated theorem as a particular case, viz., we have the theorem that, if given any positive quantity $e$ and any point $P$ in the range of the independent variable, we can find an interval $d$, the same for all the functions but depending in general on $P$, such that the oscillation of each of the functions inside an interval of length $d$ with $P$ as left (right) hand end-points is less than $e$, then the same consequences follow as in the theorem last stated.

The paper terminates with some results regarding the upper and lower bounds of successions of continuous functions.

2. Theовем 1.-If all the lower (upper) functions of an oscillating succession are lower (upper) semi-continuous, then all the upper (lower) functions of the succession are also lower (upper) semi-continuous.

For example, suppose all the lower functions to be lower semicontinuous, and take any one of the upper functions. Without any loss of generality we may, if we please, suppose it to be the upper function par excellence of the whole succession, and suppose $P$ to be a point at which it is not lower semi-continuous. Then there must be a sequence of points $P_{1}, P_{2}, \ldots$, having $P$ as limiting point, and such that the value of the upper function $u(x)$ at $P$ is greater than the limit, which we may, if we please, suppose unique, of $u(x)$ along this sequence.

Now choose, as we evidently can, a sub-set of the functions which hes the value of $u(x)$ at $P$ as unique limit at that point. Then, since the 
lower function of this sub-set is lower semi-continuous at $P$, this value is less than or equal to the limit or limits of this lower function as we move along the points $P_{1}, P_{2}, \ldots$; and therefore less than or equal to the limit or limits of the upper function of the sub-set along the same points; and therefore a fortiori less than or equal to the limit of the original upper function $u(x)$ along the same set.

But, by hypothesis, this value is greater than this latter limit, which is absurd. This proves the theorem.

Cor.-The above holds if the semi-continuity assumed is on the right (left) only, the semi-continuity proved being then of the same type.

3. Тнвовем 2.-If either all the upper, or all the lower, functions of a succession of functions, or both sets, are continuous functions, then in any sub-succession of the functions a sequence of the functions can be found, having an unique limiting function, and this limiting function is continuous.

Suppose all the upper functions are continuous.

Take any countable set of points dense everywhere, $P_{1}, P_{2}, \ldots, P_{n}, \ldots$. Select any one of the limits at $P_{1}$; we can then choose out a sub-set of the functions having, for the value of $x$ corresponding to $P_{1}$, this selected limit as unique limit. Omitting now the first function $f_{1_{1},}(x)$ of this subset, let us, in like manner, choose out a sub-set of the remaining succession, having at $P_{2}$ an unique limit, which we select to be the value at $P_{2}$ of the lower function of the first sub-set. We then repeat this process, that is, we omit the first function $f_{2,1}(x)$ of this new succession, and determine a sub-set of the remaining succession having at $P_{3}$ an unique limit, which we select to be the value at $P_{3}$ of the lower function of the second sub-set. Proceeding thus ad infinitum, we take the succession

$$
f_{1,1}(x), f_{2,1}(x), \ldots, f_{n, 1}(x), \ldots,
$$

formed by the successive omitted first members. As a sub-set of the first sub-set, this succession has an unique limit at $P_{1}$. As all but its first member belong to the second sub-set, it has an unique limit at $P_{2}$, and so on for the remaining points $P_{3}, \ldots, P_{n}, \ldots$. But these points are dense everywhere, so that the values of these unique limits determine at most one continuous function having those values at those points; this is, of course, the upper function of the succession (1), since we know that this is continuous. The lower function of the succession (1) is, by Theorem 1, upper semi-continuous, so that its value at any point is not less than the unique limit of its values as we approach the point in question 
by means of the points $P_{n}$, that is, it is not less than the value of the upper function of the same succession at the same point. But the lower function is never greater than the upper function; hence the upper and lower functions are equal at each point, that is to say, the succession (1) has an unique limiting function, and this function is continuous.

CoR. 1.-If all the upper (lower) functions are continuous, then there is an, in general, infinite set of lower (upper) functions which are continuous.

CoR. 2.-If the unique limiting functions, which are proved in Theorem 2 to exist, all coincide, then the upper and lower functions of the whole succession coincide, so that the whole succession becomes a sequence, having a continuous function as limiting function.

For, repeating the argument in the first few lines of the proof of the theorem, we can shew that the upper and lower functions in question, each of them coincide at any assigned point we please, and therefore at every point, with this unique limiting function.

Cor. 3.-If there be a finite number of distinct limiting functions, then the succession breaks up into the same number of sequences.

Take the case of two limiting functions $f(x)$ and $g(x)$; it will be seen that the argument is quite general ; it only needs repetition to apply to any finite number of limiting functions.

Let $x$ be a point at which $f(x)$ and $g(x)$ differ, and let $2 e$ be any positive quantity less than their difference. Divide the functions of the succession into two classes, the first containing all those functions which at the point $x$ differ from $f(x)$ by less than $e$, and the second containing all the remaining functions. Then the functions of the first class differ from $g(x)$ at the point $x$ by more than $e$; therefore the first class cannot yield a sequence with $g(x)$ as limit ; hence $f(x)$ is the only possible unique limiting function of the first class, which is therefore, by Cor. 2, a sequence. Similarly, the second class is a sequence with $g(x)$ as limiting function.

Cor. 4.-The above theorem and all the previous corollaries apply equally if the continuity assumed is on the right (left) only; in this case the continuity proved is also of the same type.

4. So far no limitations have been imposed directly on the character of the functions of the succession, only those indirectly involved in the assumptions as to the upper or lower functions, or both, of the succession. Let us now suppose the functions of the succession to be continuous. 
Consider the function of the two variables $x$ and $n$ given by

$$
F(x, n)=f_{n}(x),
$$

where, of course, $n$ has only integral values. Then we call the upper double limit of $F(x, n)$ at the point $(a, \infty)$ the peak function of the succession at the point $a$, and the lower double limit the chasm function.* If the peak function is at any point equal to the upper function of the succession, we say that the succession oscillates uniformly above, and if the chasm function is equal to the lower function, we say that the succession oscillates uniformly below. If both these take place, we say the succession oscillates uniformly, or more fully, oscillates uniformly both above and below. When there is uniform oscillation above (below) the upper function is necessarily upper (lower) semi-continuous, instead of being only upper (lower) semi-continuous at all points except those of a set of the first category, which is the general case.

Tests for uniform oscillation, analogous to those for uniform convergence, have been devised. $\dagger$

It may happen that a succession that oscillates uniformly above (below) is such that every sub-succession without exception has the same property; we may then say that the succession oscillates uniformly and homogeneously above (below). It follows from Theorem 1 that, in this case, if the oscillation is uniform and homogeneous above, all the lower as well as all the upper functions are upper semi-continuous, and that, if the oscillation is uniform and homogeneous below, all the upper as well as all the lower functions are lower semi-continuous. It hence follows further that, if the oscillation is uniform and homogeneous both above and below,

* When we distinguish right from left, we, of course, do so by confining $x$ io be always greater or always less than $a$. That the above is equivalent to the original definition which I gave in the present Proceedings, Ser. 2, Vol. 6, p. 300, may be shewn as follows:-

Let $U$ be this upper double limit when $a<x$, and $f_{1}\left(x_{1}\right), f_{3}\left(x_{2}\right), \ldots, f_{11}\left(x_{n}\right), \ldots$ a sequence of values having $U$ as unique limit, while $x_{1}, x_{2}, \ldots, x_{n}, \ldots$ have $a$ as unique limiting point.

Take any interval $P Q$ on the right of $P$; then, if $n$ is sufficiently large, all the points $x_{n}$ lie inside this interval ; hence, denoting as usual by $M_{n, Q}$ the upper bound of $f_{n}(x)$ in this interval, and by $M_{Q}$ the upper limit of $M_{n, Q}$ as $n$ increases indefinitely, we have

and therefore

$$
M_{n, Q} \geqslant f_{n}\left(x_{n}\right) \text {; }
$$

$$
M_{Q} \geqslant U \text {. }
$$

Letting $Q$ move up to $P$, we get, by the original definition of the right-hand peak function,

$$
\pi_{H}(a) \geqslant U \text {. }
$$

But, since $f_{n}(x)$ is a continuous function it assumes $M_{n, Q}$ as one of its values, so that $\pi_{n}(a)$ is a repeated limit, and therefore a double limit of $f_{n}(x)$, whence

$$
\pi_{R}(a) \leqslant U \text {. }
$$

The two last inequalities prove the equality of $\pi_{R}(a)$ and $U$.

Q.E.D.

$$
\text { † "On Uniform Oscillation," Camb. Phil. Trans., } 1909 .
$$


all the upper and all the lower functions are continuous. Hence it follows further by Theorem 2 that, in every sub-succession of functions there is a sequence of functions having a continuous function as unique limiting function. Moreover, as uniform convergence is a special case of uniform oscillation, the convergence of each such sequence to its limiting function must be uniform.

In other words, we have the following theorem :-

Theorex.-If a succession of functions oscillates uniformly and homogeneously, then, every sub-succession of the functions contains a sequence converging uniformly to a continuous function as unique limit, and all the upper and all the lower functions of the succession are continuous.

CoR.-If all the unique limiting functions to which the various sequences converge coincide, the whole succession is itself a sequence which converges uniformly.

5. It may be remarked that, so far, there has been no restriction that the functions concerned should be bounded, except where the words "convergence" and "continuous" occur. If, moreover the word "continuous" be interpreted in the extended sense, the two infinities, $+\infty$ and $-\infty$, being distinguished, and the word "convergence" be interpreted to mean, where necessary, divergence to an unique infinite linit, this implicit restriction is removed.

It should also be noticed that the definitions and statements of the preceding article apply equally if we restrict our attention to the neighbourhood of a single point, instead of to a whole interval, in so far as these statements then have any meaning.

More important than this is the fact that all the reasoning and all the results apply equally whatever be the number of variables, and equally if we substitute for the range of the variables any perfect set of points.

Finally, we may remark that in the case of a single variable, with obvious extensions in the case of more variables, we may distinguish between uniform and homogeneous oscillation on the right and on the left, and so get more general forms of our theorems involving continuity and semi-continuity on one side only.

6. We now shew that if, given any quantity $e$, however small, we can find an interval d, having the point $P$ for its left-hand extremity, such that the excess of the maximum of $f_{n}(x)$ in that interval over its value at $P$, is less than e, for all values of $n$ (so that $d$ is the same for all values of $n$ ), then the succession oscillates uniformly above on the right. 
Denote the upper right-hand peak function of the succession at $P$ [that is, the upper double limit at $P$ of $f_{n}(x)$ as $n$ increases indefinitely and $x$ always lies to the right of $P$ ] by $\pi_{R}(P)$. This can at most exceed the upper limit of $f_{n}(P)$ as $n$ increases indefinitely by $e$. Hence, if $u(P)$ be the upper function, we have

$$
\pi_{R}(P)-u(P) \leqslant e .
$$

But the left-hand side of this inequality is never negative, and $e$ is as small as we please. Therefore

$$
\pi_{R}(P)-u(P)=0 .
$$

Thus the succession oscillates uniformly above on the right at $P$. But the same argument applies to any sub-succession, whence we see that the uniform oscillation above on the right at $P$ is also homogeneous.

7. The result of the preceding article enables us to give the following theorem, which includes Arzelà's celebrated theorem as a particular case :-

Theorem.-If a succession of functions is such that, given any positive quantity $e$, and any point $P$ in the range of variation of the variable, we can find an interval d (this interval being the same for all the functions, but depending in general* on P), such that the oscillation of each of the functions inside an interval of length $d$ with $P$ as left-hand (right-hand) end-point is less than e, then (1) all the upper and all the lower functions are continuous on the right (left), and (2) there is in every sub-succession at least one sequence of the functions which converges towards an unique limiting function which is continuous on the right (left), the convergence to this limiting function being, moreover, uniform on the right (left).

This theorem is a corollary to the theorem virtually proved above, viz.,

Theorem.-If a succession of functions oscillates uniformly and homogeneously on the right (left), then all the upper and all the lower functions are continuous on the right (left), and in every sub-succession there is a sequence of the functions converging towards an unique limiting function which is continuous on the right (left), the convergence to this limiting function being, moreover, uniform on the right (left).

8. It should be remarked that the difference between the alternatives in the former of the two theorems in $\$ 7$, and therefore between either of them and the special case of both, including Arzeli's theorem, in which no distinction is made between right and left, has reference at most

* When we consider right and left simultaneously, we can, of course, pass from the statement for the point $P$ to that for the whole interval, by using the Heine-Borel theorem. 
to a countable set of points, as there is no distinction of right and left as regards both discontinuities of a function or uniformity of oscillation except at a countable set of points.

It is also worthy of notice that the condition of uniform and homogeneous oscillation appears to involve fewer restrictions than Arzela's assumption of equal continuity. This is not in contradiction with Arzelà's statement of his theorem which gives a sufficient condition that in a bounded succession of functions there should be at least one sequence converging uniformly, while it asserts that the condition is necessary only when the given succession is a sequence.

9. One of the most remarkable cases where we actually obtain successions of the kind contemplated is when integrating other successions. The results arrived at above enable us to go some steps further in some of the theorems stated and proved in my paper on "Term-by-Term Integration of Oscillating Series."* Thus, in Theorem 7, p. 113, of that paper, the restriction that the upper function $u(x)$ must have a finite integral may now be removed, and the first of the statements on p. 110 takes the more general form :-

If the point $P$ be such that, in its right-hand neighbourhood $s_{n}(x)$ regarded as a function of $(x, n)$ is bounded above, then the integral series oscillates uniformly and homogeneously above on the right, and below on the left, at $P$, so that, moreover, all the upper functions and all the lower functions are upper semi-continuous on the right and lower semi-continuous on the left at $P$.

We get, of course, similar statements when we substitute "left" for "right," and also if we substitute "lower" and "below" for "upper" and "above." In particular, if $s_{n}(x)$ is bounded above throughout the whole interval, all the upper and all the lower functions of the integral series are upper semi-continuous on the right and lower semi-continuous on the left, the integral series oscillating everywhere uniformly and homogeneously above on the right, and uniformly and homogeneously below on the left, and a corresponding statement holds if $s_{n}(x)$ is bounded below. If $s_{n}(x)$ is bounded both above and below, we can add nothing to the statements already made in the paper in question, except that it should be noticed that the results of the present paper with regard to the existence of sequences in the succession apply to the succession of integrals. This case has been virtually considered by 
Arzelà, loc. cit., for he considers successions of functions whose derivates are in their ensemble bounded. Interpreting, therefore, integration in the generalised sense unknown to Arzeli at the time of writing his paper, the identity of his series in essentials with mine becomes evident. Formally, however, there is still a difference, for my bounded functions are not necessarily derivates; they may be any whatever. The upper and lower derivates of the Lebesgue integral of a bounded function lie between the bounds of the function and therefore differ from these, which are also bounded, only at a set of points of content zero (cp. also $\$ 8$, p. 107, of "Term-by-Term Integration of Oscillating Series"). Hence the fact stated follows.

The following is an alternative proof of this theorem :-

$$
S_{n_{i}}\left(x_{n_{i}}\right)=\int_{a}^{x_{n_{i}}} s_{n_{i}}(t) d t=\int_{a}^{x} s_{n_{i}}(t) d t+\int_{x}^{x_{n_{i}}} s_{n_{i}}(t) d t \leqslant S_{n_{i}}(x)+M_{x}\left(x_{n_{i}}-x\right),
$$

supposing $x_{n_{\mathrm{i}}}$ to lie to the right of $x$, and $M_{x}$ to be the finite upper bound of $s_{n}(x)$ regarded as a function of $n$ and $x$ in some interval to the right of $x$ inside which $x_{n_{i}}$ lies.

Now, since the peak function of the integrated succession $S_{1}, S_{2}, \ldots$ is the upper double limit of $S_{n}(t)$ at $t=x, n=\infty$, we can choose such a sequence of integers $n_{i}$ and corresponding points $x_{i_{i}}$ with $x$ as limiting point, that $S_{n_{i}}$ has the right-band peak function $\mathrm{II}_{R}(x)$ as unique limit. The first term on the right then has a limit, or limits, $\leqslant U(x)$, the upper function of the integrated succession, and the second term the limit zero; hence

$$
\Pi_{R}(x) \leqslant U(x),
$$

which, since the upper function is never greater than the peak function, gives

$$
\Pi_{R}(x)=U(x),
$$

shewing that the integrated succession oscillates uniformly above on the right at the point $x$.

Q.E.D.

Similarly, if $x_{n_{\mathrm{i}}}$ lies on the left of $x$, we have

$$
S_{n_{\mathrm{i}}}\left(x_{n_{\mathrm{i}}}\right)+\int_{x_{n_{i}}}^{x} s_{n_{i}}(t) d t=S_{n_{\mathrm{i}}}(x)
$$

whence, by the above argument, using "less" instead of "greater,"

$$
X_{L}(x)=L(x),
$$

shewing that the integrated succession oscillates uniformly below on the left at the point $x$.

Q.E.D. 
Since the property that $s_{n}(x)$ has of being bounded above involves the same property for every sub-succession, it follows that the uniformity proved is also homogeneous, as stated above.

10. Another application which may be made of the theorems of the present paper is to the theory of the upper and lower bounds of successions of continuous functions. It is often of importance to know when the upper or lower bound of the limit of a sequence of functions is the limit of the upper or lower bound of the constituent functions of the sequence.* In the more general case of an oscillating succession, two questions present themselves :-

(1) Is the upper (lower) bound of the upper (lower) function the upper (lower) limit of the upper (lower) bounds of the constituent functions of the succession?

(2) Has the succession a limiting function, which itself possesses this property?

These questions are answered by the following theorems.

Theorex.-If the succession oscillates uniformly above (below), or, more generally, if it everywhere either oscillates uniformly above (below), or has a non-uniformity of the upper (lower) oscillation which is completely visible, then the upper (lower) limit of the upper (lower) bounds of the succession is the upper (lower) bound of the upper (lover) function.

I have already in a previous paper explained the difference between a visible and an invisible point of non-uniform oscillation of a succession of continuous functions. The non-uniformity of the upper oscillation is said to be invisible if the point is one at which the upper function is upper semi-continuous. I here use the expression "completely visible" to denote that the peak function is the upper limit of the upper function at the point in question.

To prove this theorem, take a sequence of values of $n$, for which the upper bounds of the corresponding functions $f_{n}(x)$ have for unique limit the upper limit $U$ of all the upper bounds. The points at which the upper bounds of these selected functions are assumed as values by those functions will have one or more limiting points. At each such limiting point $x$, the peak function is evidently equal to this limit $U$. Hence there is at least one point at which the upper function, if the oscillation at that point is uniform above, or the associated upper limiting function of the upper function if the non-uniformity of the upper oscillation be every-

" "On Functions Defined by Monotone Sequences and their Upper and Lower Bounds," Messenger of Wath. (1908), pp. 148-154. 
where completely visible, assumes the value $U$. This proves the theorem, since it is evident that the upper bound of the upper function cannot be greater than $U$.

It by no means follows, however, that there is any unique limiting function which has this value for upper bound. To be sure that we get an unique limiting function in every sub-succession we must, if we are to apply the theorems of the present paper, assume that the upper functions and the lower functions are at least continuous on one side, say on the right. This will, as we have seen, be insured, if we require the whole succession to oscillate uniformly and homogeneously on the right. In this case, then, we could assert the existence of a function which for some sub-succession is the unique limiting function, and whose upper bound is the limit of the upper bounds of the constituent functions of the original succession. We have accordingly the following theorem :-

Theorem.-If the succession oscillates uniformly and homogeneously above on the right, and it has a non-uniformity above on the left which, wherever it occurs, is completely visible, and where there is uniformity above on the left, this is not necessarily homogeneous, then the succession contains a sequence of the functions the upper bound of whose unique limiting function is the upper limit of the upper bounds of the whole succession of functions.

If we attempt to get a more symmetrical theorem, we have to hypothecate that the upper and lower functions are all continuous. In this case there can be no non-uniformity, for there is evidently no visible non-uniformity. The theorem now takes the comparatively special form :-

If the succession oscillates uniformly and homogeneously both above and below, then there is an unique limiting function of some subsequence of the functions which assumes both the upper limit of the upper bounds and the lower limit of the lower bounds of all the constituent functions of the succession.

This last result corresponds precisely to that obtained by Arzeli, except that he requires the apparently more stringent condition of equal continuity of the succession of functions.

It may be noted finally that from the first theorem of the present article the result of the paper quoted "On Monotone Sequences," in the special case in which the constituent functions are continuous, may be deduced. In a monotone sequence, in fact, the non-uniformity of the convergence is always completely visible. 
11. When the points of non-uniform oscillation are invisible, or only partially visible, it is evident that the argument used in the preceding article fails. Even when the succession of functions consists of a sequence of continuous functions having a continuous function as unique limiting function, it by no means follows that either the upper bounds or the lower bounds of the constituent functions have an unique limit, still less that they have unique limits which are respectively the upper and lower bounds of the unique limiting function. All that we can assert is that the upper bound of the limiting function is not greater than any one of the limits of the upper bounds, and that the lower bound of the former is not less than any of the limits of the lower bounds.

An interesting and important case of this in practice occurs when we have a function of $(x, y)$, continuous with respect to $x$, and continuous with respect to $y$, but not continuous with respect to $(x, y)$, and we are concerned with the maxima (minima) of the function for fixed values of $y$. These form, of course, a function of $y$, the maxima forming a lower semicontinuous and the minima an upper semi-continuous function of $\mathrm{y}$. We cannot assert the continuity of these functions unless $f(x, y)$ is a continuous function of $(x, y)$. In the contrary case these functions, far from being continuous, are not even necessarily bounded.*

* For further developments of the theory of oscillating successions of functions, see a forthcoming paper by the author cntitled " On Semi-Integrals and Successions of Functions." 